\title{
Computational prediction and characterisation of miRNAs and their pathway genes in human schistosomiasis caused by Schistosoma haematobium
}

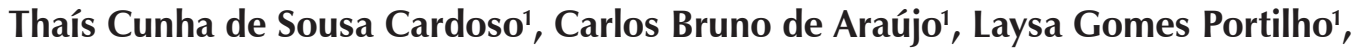 \\ Luiz Guilherme Alves Mendes', Tamires Caixeta Alves', Gustavo Caetano Silva', \\ Thales Henrique Cherubino Ribeiro², Peterson Elizandro Gandolfi ${ }^{1,3}$, Enyara Rezende Morais ${ }^{1,3}$, \\ Laurence Rodrigues do Amaral ${ }^{1,3}$, Matheus de Souza Gomes ${ }^{1,3 /+}$ \\ Universidade Federal de Uberlândia, Laboratório de Bioinformática e Análises Moleculares, Patos de Minas, MG, Brasil \\ ${ }^{2}$ Universidade Federal de Lavras, Departamento de Biologia, Setor de Fisiologia Vegetal, Laboratório de Fisiologia Molecular de Plantas, \\ Lavras, MG, Brasil \\ ${ }^{3}$ Universidade Federal de Uberlândia, Rede Multidisciplinar de Pesquisa, Ciência e Tecnologia, Patos de Minas, MG, Brasil
}

BACKGROUND Key genes control the infectivity of the Schistosoma haematobium causing schistosomiasis. A method for understanding the regulation of these genes might help in developing new disease strategies to control schistosomiasis, such as the silencing mediated by microRNAs (miRNAs). The miRNAs have been studied in schistosome species and they play important roles in the post-transcriptional regulation of genes, and in parasite-host interactions. However, genome-wide identification and characterisation of novel miRNAs and their pathway genes and their gene expression have not been explored deeply in the genome and transcriptome of $S$. haematobium.

OBJECTIVES Identify and characterise mature and precursor miRNAs and their pathway genes in the S. haematobium genome.

METHODS Computational prediction and characterisation of miRNAs and genes involved in miRNA pathway from $S$. haematobium genome on SchistoDB. Conserved domain analysis was performed using PFAM and CDD databases. A robust algorithm was applied to identify mature miRNAs and their precursors. The characterisation of the precursor miRNAs was performed using RNAfold, RNAalifold and Perl scripts.

FINDINGS We identified and characterised 14 putative proteins involved in miRNA pathway including ARGONAUTE and DICER in $S$. haematobium. Besides that, 149 mature miRNAs and 131 precursor miRNAs were identified in the genome including novel miRNAs.

MAIN CONCLUSIONS miRNA pathway occurs in the $S$. haematobium, including endogenous miRNAs and miRNA pathway components, suggesting a role of this type of non-coding RNAs in gene regulation in the parasite. The results found in this work will open up a new avenue for studying miRNAs in the $S$. haematobium biology in helping to understand the mechanism of gene silencing in the human parasite Schistosome.

Key words: human parasite - small RNAs - bioinformatics - neglected diseases

The human schistosomiasis is a parasitic, chronic and debilitating disease caused by helminths of the Schistosoma genus that currently affects about 240 million people worldwide, according to the World Health Organization. ${ }^{(1)}$ The countries of Africa and South America are among the most affected. In addition, 800 million people live in areas at risk of contracting the disease. ${ }^{(2)}$ Five Schistosoma species are responsible for most of human infections: Schistosoma mansoni, Schistosoma japonicum, S. haematobium, Schistosoma mekongi and Schistosoma intercalatum. ${ }^{(1)}$

$S$. haematobium affects the reproductive and urinary system and it has been considered a major public health problem mainly in Africa. ${ }^{(1,3)}$ The infection by this parasite

doi: 10.1590/0074-02760190378

TCSC, CBA and LGP contributed equally to this work.

+ Corresponding author: matheusgomes@ufu.br

(D) http://orcid.org/0000-0001-7352-3089

Received 14 October 2019

Accepted 03 March 2020 can results in nutritional deficiencies and growth retardation, may also lead to a decrease in the cognitive system. (4) The spread of this disease occurs by fresh water and by the presence of host snails. Activities in water contact, such as agriculture, can facilitate the parasite infection. ${ }^{(3)}$

The dissemination of the disease is dependent of interaction between parasite and intermediate host. In S. mansoni and their intermediate host Biomphalaria glabrata, the interaction is complex and determined by some genes involved in the parasite infectivity and host susceptibility. ${ }^{(5,6)}$ Thus, it is also suggested a complex host/parasite interation involving several genes between $S$. haematobium and its intermediate snail host, from the genus Bulinus. Therefore, understanding the mechanisms of gene regulation is critically important for understanding the host-parasite interactions and which genes might contribute to the infection.

The regulation of gene expression in eukaryotes comprises processes involved in translation repression and/or degradation of target genes. ${ }^{(7)}$ Small RNAs, such as microRNAs (miRNAs), and their silencing pathways 
have been considered important in several organisms by performing a fine and specific regulation of gene expression maintaining genome integrity. ${ }^{(7,8)}$

The miRNAs are small non-coding endogenous RNAs with about 22 nucleotides in length of acting posttranscriptionally typically via imperfect complementary base pairing binding to the target genes. ${ }^{(7,9,10)}$ To identify mature miRNAs and their precursors several studies have been using experimental and computational strategies. The experimental approaches, despite presenting physical evidence of the miRNAs presence, can exclude molecules expressed in certain stages and tissues. ${ }^{(10,11,12)}$ Thus, in silico analysis are useful for species with complete or incomplete sequenced genomes, allowing the discovery of new miRNAs using whole-genome DNA information. ${ }^{(11)}$

The genes involved in gene silencing pathway mediated by miRNAs have been described in several model organisms such as Drosophila melanogaster, Caenorhabditis elegans and Homo sapiens. ${ }^{(13,14)}$ In addition, members of the biosynthetic pathway of miRNAs, such as ARGONAUTE and DICER, as well as miRNA molecules, have been reported in some Schistosoma species, including $S$. mansoni, S. japonicum and S. haematobium. ${ }^{(15)}$

Young et al., published in 2012 the whole-genome sequence of S. haematobium. ${ }^{(16)}$ The interpretation of schistosome draft genomes has improved our understanding of the molecular biology of these parasites allowing the identification and characterisation of undiscovered genes. In the face of the vastly increased importance of such non-coding RNAs in the gene expression regulation, it is important that we study mature and precursor miRNAs, as well as the genes involved in miRNA pathway in this $S$. haematobium. This might help to understand the life cycle of this parasite, its infectivity mechanism and for searching new methods of schistosomiasis control. In this work, we identified and characterised the miRNA pathway genes, the mature miRNAs and their precursors in the $S$. haematobium genome. The results found in this work will open up a new avenue for studying miRNAs in the $S$. haematobium biology in helping to understand the mechanism of gene silencing in the human parasite Schistosome.

\section{MATERIALS AND METHODS}

Prediction and characterisation of genes involved in the miRNAs pathway - Firstly, to search the putative protein sequences involved in the miRNA pathway in Schistosoma haematobium, BLASTp tool was used (National Centre for Biotechnology Information - http://www. ncbi.nlm.nih.gov/) using as queries sequence reference proteins from animal species, such as D. melanogaster and $C$. elegans, model organisms. The putative protein sequences in $S$. haematobium were found and collected from SchistoDB, S. haematobium Egypt genome (v 44, 2019-06-24) (http://schistodb.net). To improve the annotation of these predicted proteins involved in the miRNA pathway in S. haematobium, other gene prediction technique was used. The $S$. haematobium Egypt genome (v 44, 2019-06-24) was retrieved from the Schistosoma Genomic Resource database. To improve gene prediction of the putative genes involved in miRNA pathway in
S. haematobium genome, single-end reads from a single cDNA library of $S$. mansoni were retrieved from SRA accession SRR629229. After quality control, approximately $63 \%$ of 36.836 .649 reads were successfully mapped to the S. haematobium Egypt genome with STAR (v 2.5.3ab) using the parameter twopassMode. ${ }^{(17)}$ The introns coordinates from this alignment were converted to GFF format to train GeneMark-ET algorithm (v 4.33). ${ }^{(18)}$ In this way 19.686 putative genes were predicted. In addition, GeneMark-ES (v 4.33) was also applied to S. haematobium Egypt genome to predict genes without RNA-seq support, 18.622 putative genes were predicted with this approach. To identify the orthologous genes, BLAT (v 35 ) was applied to search query proteins against the reference genome. ${ }^{(19)}$ The top 10 hits of each query were manually compared against the two sets of predicted genes using the Integrated Genome Browser (v 9.0.0). ${ }^{(20)}$ In addition, Augustus web tool (http://augustus.gobics.de/) was used to improve gene prediction using the genomic locus around the BLAT results, setting the parameters to find similarities with $S$. mansoni genes. The consensus sequences, from the BLAT alignment, and the predicted genes were then aligned to their orthologous using CLUSTALX (v 2.1) (http://www.clustal.org/) and finally subjected to conserved domains searches with PFAM online tool (v 31.0) (http://pfam.xfam.org/).

The characterisation and in silico validation of the putative protein sequences involved in the miRNA pathway in S. haematobium were performed using the position and the presence of the conserved domains and also of the amino acids from active sites using PFAM and Conserved Domains Database (CDD) (http://www.ncbi. nlm.nih.gov/cdd/).

Expression analysis - The transcripts of the genes involved in the miRNA pathway in S. haematobium were individually analysed using RNASeq data set, retrieved from NCBI Sequence Read Archive - SRA, of three different developmental stages of S. haematobium: adult female (SRR6655495), adult male (SRR6655497) and egg (SRR6655493). ${ }^{(16)}$ Quality control and adapter removal were conducted using Trimmomatic (v 0.36). Single-end reads were aligned against pre-selected sequences using bowtie2 (v 2.3.0) with the "--very-sensitive-local parameter". Alignment sam files were sorted and converted to bam files with samtools (v 1.6). Expression values were extracted from the alignment results using express (v 1.5.1) and RPKM (Reads Per Kilobase of transcript, per Million mapped reads) were calculated after library size normalisation with the Bioconductor package edgeR in the R statistical environment. The results of the expression analysis were plotted in a heatmap, where the colour system applied comprises an intense red colour as the highest transcript expression and an intense blue colour as the lowest transcript expression.

Prediction and characterisation of mature miRNAs and their precursors (pre-miRNAs) - A robust algorithm used to predict miRNAs in $S$. mansoni genome ${ }^{(10)}$ was also used for identification and characterisation of mature miRNAs and their precursors in S. haematobium Egypt genome. The data was applied to Einverted (EM- 
BOSS tool) ${ }^{(21)}$ and BLASTN programs (http://www.ncbi. nlm.nih.gov/) to select sequences that show potential hairpin formation or similarities with precursor miRNAs. These sequences were applied in a set of filters accepting in the end of the algorithm putative real conserved miRNAs. These filters were based on conserved characteristics of precursor miRNAs and regions that have no potential formation of pre-miRNAs were discarded. The filters used were: guanine and cytosine (GC content), minimal energy free (MFE), homology with mature miRNAs already identified, homology with protein coding region, homology to repetitive regions and homology with non-coding RNAs.

The miRNA cluster searching was optimised using information obtained from the position (start and the end position of the precursor miRNAs) each miRNA identified in the S. haematobium Egypt genome. miRNAs found at a distance of less than $10 \mathrm{k}$ nucleotides downstream or upstream depending on the direction in the DNA strand were considered linked in a specific miRNA cluster. Each sequence portion was retrieved from the S. haematobium Egypt genome using information from the first and last nucleotides of the cluster. The secondary structure of each cluster was obtained using the RNAfold tool (http://rna.tbi.univie.ac.at/cgi-bin/ RNAWebSuite/RNAfold.cgi).

miRNA sequences identified as putative real conserved miRNA precursors in S. haematobium genome were characterised structurally and thermodynamically using RNAfold, RNAalifold (Vienna RNA Package) (http://rna.tbi.univie.ac.at/cgi-bin/RNAWebSuite/ RNAalifold.cgi) and homemade Perl scripts. ${ }^{(22)}$

miRNAs in S. haematobium Egypt small RNAseq libraries - Two small RNA data files of adult male (SRR6655496) and adult female worms (SRR6655494) were retrieved from the NCBI Sequence Read Archive (SRA). The library qualities were evaluated using FastQC software. ${ }^{(23)}$ The adapters were removed with Trimmomatic ${ }^{(24)}$ discarding reads with quality score below 20 and length less than 17 nucleotides and longer than 30 nucleotides. The filtered sequences were mapped and quantified using miRDeep2. ${ }^{(25)}$ miRDeep2 and perl scripts were used on each sequence separately to generate the numbers of the reads for each miRNAs identified.

Sequence alignments and phylogenetic analysis - The multiple sequence alignment (MSA) were performed to S. haematobium Egypt putative proteins involved in the miRNA pathway and also for the S. haematobium Egypt pre-miRNA sequences and their respective orthologs. These analyses were performed by CLUSTALX 2.0 with default parameters multiple sequence alignment of proteins and for the pre-miRNA sequences were used the following adjusted parameters: gap opening 22.50 and gap extension 0.83 .

Phylogenetic analysis was performed using MEGA version $\mathrm{X}^{(26)}$ and Neighbor-Joining. ${ }^{(27)} \mathrm{A}$ consensus tree was generated applying a bootstrap of 5.000 replicates for pre-miRNA sequences and 2.000 replicates for protein sequences. The calculation of their evolutionary distance was performed using Kimura-2-parameters for the pre-miRNA and JTT for protein sequences. ${ }^{(27)}$
Statistical analysis - For the statistical comparisons among the structural and thermodynamic variables of each category (superphylum, phylum and/or genus), a basic descriptive analysis was performed followed by non-parametric tests (Wilcoxon-Mann-Whitney). Median values were used to perform the statistical comparisons. ${ }^{(28)}$ Statistical significance was set at $p<0.05$.

\section{RESULTS}

Identification, characterisation, phylogenetic analysis of putative proteins involved in miRNA pathway - The predicted proteome version 44 of the S. haematobium genome obtained from SchistoDB was used to identify and characterise the predicted $S$. haematobium proteins involved in the miRNA pathway. Fourteen putative $S$. haematobium proteins involved in the miRNA pathway were identified and characterised according to their putative function based on homology, conserved domain distribution (and conservation) and protein size. These $S$. haematobium proteins were compared with their miRNA pathway orthologue proteins from related species such as Schistosoma species and model species (e.g. C. elegans and D. melanogaster) (Table). In order to confirm and improve the prediction of the proteome version 44 of the $S$. haematobium an alternative predicted genome-based proteome was performed using information from the $S$. haematobium genome and RNAseq sequences (SRR6655495, SRR6655497, SRR6655493). 18.622 putative genes were predicted with this alternative approach to predict the $S$. haematobium proteome. Comparing the proteins involved in the miRNA pathway predicted from $S$. haematobium version 44 to proteins obtained from the alternative predicted proteome only the DROSHA protein sequence was divergent. The putative DROSHA sequence predicted from proteome version 44 - SchistoDB showed 1041 amino acids in length and only one Ribonuclease III conserved domain. The new prediction of DROSHA showed a protein with two Ribonuclease III and one DSRM conserved domains with 1581 amino acids in length corroborating with their orthologs and thus used for further analysis. Among the fourteen putative S. haematobium proteins involved in the miRNA pathway identified, two key protein families of the canonical miRNA biogenesis pathway were considered for the further analysis: ARGONAUTE and RNAse III proteins (DICER and DROSHA). We were able to identify and characterise in S. haematobium data and considered for the further analysis two AGO proteins (sht_Ago_MS3 08447/ sht Ago MS3 01142), two DICER proteins (sht Dcr MS3_05083 and sht_Dcr_MS3_09247) and one DROSHA protein (sht_Drsh_M̄MS3_06910).

ARGONAUTE - The putative sht_Ago_MS3_08447 protein displayed only the PIWI and PAZ domains at positions 559-852 and 304-400, respectively. On the other hand, the putative protein sht_Ago_MS3_01142 showed the PAZ, PIWI, ARGOL1, ARGOL 2, ARGON and ARGOMID domains. These $S$. haematobium Ago proteins and the distribution of their conserved domains corroborated with their orthologues in S. mansoni (Smp) and $S$. japonicum (Sjp) ${ }^{(15)}$ (Fig. 1). 
TABLE

Putative proteins involved in miRNA pathway identified in Schistosoma haematobium data compared with their orthologs from Schistosoma mansoni and Schistosoma japonicum

\begin{tabular}{|c|c|c|c|c|c|c|c|c|}
\hline $\begin{array}{l}\text { ID protein } \\
\text { S. haematobium }\end{array}$ & Putative name & $\begin{array}{l}\text { Length } \\
\text { (aa) }\end{array}$ & $\begin{array}{l}\text { ID protein } \\
\text { S. mansoni }\end{array}$ & E-value & $\begin{array}{l}\text { Length } \\
\text { (aa) }\end{array}$ & $\begin{array}{c}\text { ID protein } \\
\text { S. japonicum }\end{array}$ & E-value & $\begin{array}{l}\text { Length } \\
\text { (aa) }\end{array}$ \\
\hline \multirow{3}{*}{ MS3_01142 } & \multirow{3}{*}{ Sht_Argonaute } & \multirow{3}{*}{1009} & Smp_198380.1 & 0.0 & 928 & Sjp_0044720 & 0.0 & 987 \\
\hline & & & Smp_102690.1 & $4 e^{-105}$ & 783 & Sjp_0103990 & $2 \mathrm{e}^{-106}$ & 904 \\
\hline & & & Smp_179320.2 & $1 \mathrm{e}^{-118}$ & 921 & Sjp_0045200 & $2 \mathrm{e}^{-115}$ & 924 \\
\hline \multirow{3}{*}{ MS3_08447 } & \multirow{3}{*}{ Sht_Argonaute } & \multirow{3}{*}{882} & Smp_198380.1 & $5 \mathrm{e}^{-101}$ & 928 & Sjp_0044720 & $7 \mathrm{e}^{-101}$ & 987 \\
\hline & & & Smp_102690.1 & 0.0 & 783 & Sjp_0103990 & 0.0 & 904 \\
\hline & & & Smp_179320.2 & 0.0 & 921 & Sjp_0045200 & 0.0 & 924 \\
\hline MS3_06910 & Sht_Drosha & 1581 & Smp_142510.1 & 0.0 & 1531 & Sjp_0048900.1 & 0.0 & 1611 \\
\hline \multirow{2}{*}{ MS3_05083 } & \multirow{2}{*}{ Sht_Dicer } & \multirow{2}{*}{929} & Smp_169750.2 & 0.0 & 2485 & Sjp_0069770 & $4.2 \mathrm{e}^{-129}$ & 2480 \\
\hline & & & XP_018644375.1 & 0.0 & 2319 & - & - & - \\
\hline MS3_09247 & Sht_Dicer & 2588 & Smp_033600 & $5 \mathrm{e}^{-17}$ & 954 & Sjp_0043700 & $2 e^{-17}$ & 923 \\
\hline MS3_10205 & Sht_Exportin 5 & 611 & Smp_137650 & 0.0 & 1164 & Sjp_0006510 & 0.0 & 695 \\
\hline MS3_11315 & Sht_TSN & 1162 & Smp_246840 & $8.4 \mathrm{e}^{-116}$ & 992 & Sjp_0048060 & $5 \mathrm{e}^{-165}$ & 1428 \\
\hline MS3_03052 & Sht_Logs & 284 & Smp_023670 & 0.0 & 356 & Sjp_0045700 & $6 \mathrm{e}^{-178}$ & 369 \\
\hline MS3_05484 & Sht_FRX1 & 339 & Smp_099630 & 0.0 & 598 & Sjp_0017160 & 0.0 & 598 \\
\hline MS3_06671 & Sht_Exportin-1 & 1031 & Smp_124820 & 0.0 & 828 & Sjp_0069890 & 0.0 & 1128 \\
\hline MS3_07205 & Sht_TSN-a & 718 & Smp_166110 & $8 e^{-82}$ & 378 & Sjp_0100020 & 0.0 & 971 \\
\hline MS3_08368 & Sht_DGCR8 & 298 & Smp_087220 & 0.0 & 760 & Sjp_0013270 & 0.0 & 760 \\
\hline MS3_10949 & Sht_PASHA & 395 & Smp_087220 & 0.0 & 760 & Sjp_0013270 & $2 \mathrm{e}^{-129}$ & 760 \\
\hline MS3_11239 & Sht_VIG & 237 & Smp_009310 & $2 \mathrm{e}^{-121}$ & 417 & Sjp_0074950 & $5 \mathrm{e}^{-97}$ & 414 \\
\hline
\end{tabular}

The PIWI domain of these proteins showed a catalytic triad formed by DDH (aspartic acid, aspartic acid and histidine). In sht_Ago_MS3 08447, the catalytic triad was found at positions Asp621, Asp711 and His844. In sht Ago MS3 01142, the same triad was identified at positions Asp747, Asp819 and His957. Besides that, this triad showed high conservation in relation to ortholog AGO proteins (Fig. 2).

The AGO protein family can be divided into two subfamilies, AGO and PIWI. AGO and PIWI orthologous proteins from animal species were used to compare with the putative $S$. haematobium AGO proteins. The organisation of the AGO protein family clades in the phylogenetic analysis occurred as expected, in distinct clades, AGO subfamily grouped with AGO orthologous proteins including $S$. haematobium AGO proteins and PIWI subfamily with PIWI orthologous proteins. The distribution of the AGO and PIWI proteins from animal species corroborate with the species distribution in the tree of life (Fig. 3). We observed that S. haematobium putative proteins showed a suitable organisation compared with their orthologs, in the Plathyhelminthe clade, with S. haematobium, S. mansoni and S. japonicum proteins clustering closest within the clade.

DICER and DROSHA - Two DICER proteins (sht Dcr_MS3_05083 and sht_Dcr_MS3_09247) and one DRŌSHA ${ }^{-}$protein (sht_Drsh_MST3_06̄910) were identi- fied and characterised. The sht_Dcr_MS3 05083 displayed four domains, while sht_Der_MS3_09247 two conserved domains, and sht_Drsh_MS3_06910 three conserved domains. The putative sht Der_MS3 05083 protein identified in the S. haematobium Egypt genome presented, in its structure, the conserved domains Dicer Dimer, PAZ, Ribonuclease III (Riboc I), Ribonuclease III (Riboc II), in the positions 896-1005, 1336-1508, 1926-2102, 2212-2474, respectively. The putative sht Dcr_MS3_09247 presented only the Ribonuclease III (Riboc I) and Ribonuclease III (Riboc II) domains, in the positions 548-659 and 801-889. Finally, the putative sht_Drsh_MS3_06910 showed the Ribonuclease III (Riboc I), Rībonuclease III (Riboc II) and DSRM domains, at positions 941-1002, 1076-1229, 1258-1327, respectively, in its structure (Fig. 4).

The two Ribonuclease III conserved domains identified in the putative DICER proteins (sht_Dcr MS3_05083 and sht_Dcr_MS3_09247), showed an active site high conserved compared to their orthologs. Both domains displayed the conserved active site catalytic motif (EDDE): glutamic acid (E), aspartate (D), aspartate (D) and glutamic acid (E). The domains Riboc I in the sht_Dcr_MS3_05083 presented the catalytic residues conserve-d, at positions Glu2215, Asp2219, Asp2280 and Glu2283 and in sht_Dcr_MS3_09247 at positions Glu551, Asp555, Asp645 and Glu648. In the 


\section{Sht MS3 08447 Schistosoma haematobium}

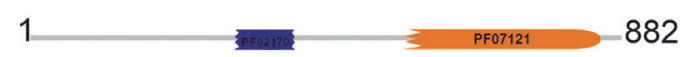

Smp 198380.1 Schistosoma mansoni

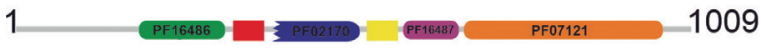

Smp 179320.2 Schistosoma mansoni

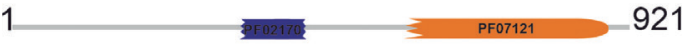

Smp 102690.1 Schistosoma mansoni

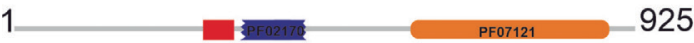

Sjp 0103990 Schistosoma japonicum

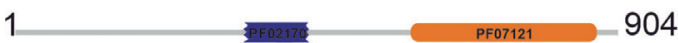

Sjp 0045200 Schistosoma japonicum

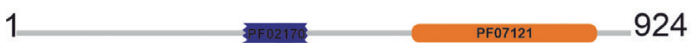

Sjp 0044720 Schistosoma japonicum

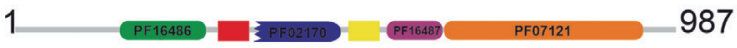

NP 493837.1 Caenorhabditis elegans

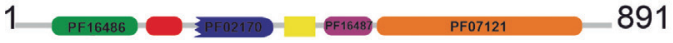

NP 001261882.1 Drosophila melanogaster
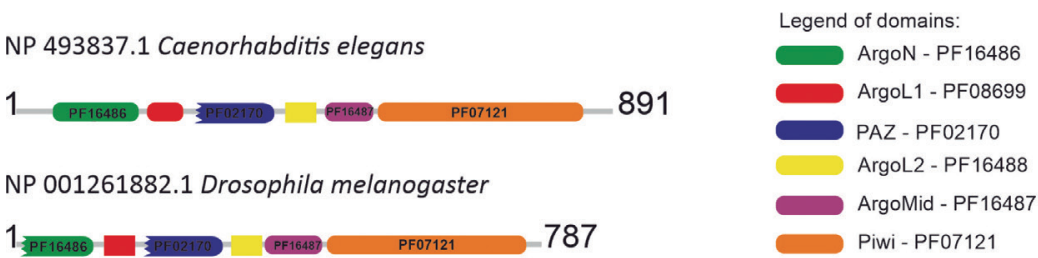

Fig. 1: conserved domains found in AGO proteins of Schistosoma haematobium Egypt versus their orthologous from S. mansoni and S. japonicum, and model organisms Caenorhabditis elegans and Drosophila melanogaster.

putative protein sht Drs MS3 06910 the catalytic residues of Riboc I domain showed in the positions Glu944, Asp948, Asp1017 and Glu1020 (Fig. 5).

The phylogenetic analysis of DICER and DROSHA putative proteins displayed the evolutionary relationship with their orthologs and paralogues. The S. haematobium proteins clustered within Platyhelminthes clade closest to $S$. mansoni and $S$. japonicum DICER and DROSHA proteins. The phylogenetic tree displayed two distinct clades (DICER and DROSHA clades) corroborating with the literature and the distribution of the species in the tree of life (Fig. 6).

Expression analysis of miRNA pathway transcripts - The genes involved in miRNA pathway identified in the genome data of $S$. haematobium were used to perform the expression analysis. This procedure occurred through submitting these transcripts individually to analyse against RNASeq data set for a library of three different stages of development in S. haematobium, adult female (SRR6655495), adult male (SRR6655497) and egg (SRR6655493). The heatmap showed the expression profile of the transcripts in the egg, adult female and adult male stages of the $S$. haematobium [Supplementary data I (Fig. 1)]. The gene that showed a highest expression level was the VIG (MS3_11239) that showed a more intense red colour in the heatmap for the three stages presented. The transcript of FMR1 (MS3_05484) also showed high level of expression in the three stages. The Expol transcript (MS3_06671) presented a high expression in egg and adult male, and a medium expression in adult female. The TSNA transcript (ID MS3 07205) presented a high expression in egg and adult female and a medium expression in adult male. The transcripts of the putative ARGONAUTE (sht_Ago_MS3_08447), showed a high level of expression in adult female and egg, and a low expression in the adult male. The others transcripts ARGONAUTE (sht_Ago_MS3 01142), DROSHA (sht_Drsh_MS3 06910) and DICER (sht Dcr_MS3_05083 and sht_Dēcr_MS3_09247), showed $\bar{a}$ low expression in all stages.

Identification and characterisation of precursor and mature miRNAs in S. haematobium Egypt genome - We identified in the $S$. haematobium Egypt genome 149 mature miRNAs and 131 pre-miRNAs [Supplementary data II (Table I)]. Regarding the miRNA precursor localisation in $S$. haematobium Egypt genome, 98 (73.68\%) were found in intergenic regions and 34 (26.32\%) intragenic [Supplementary data II (Table II)]. In addition, it was possible identify that 15 pre-miRNAs were organised in clusters $(10 \mathrm{~kb}$ as the maximum distance between 

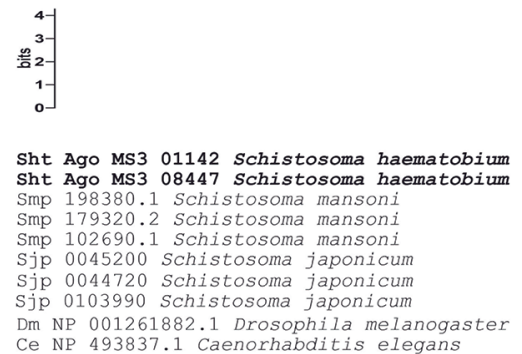

Dm NP 001261882.1 Drosophila melanogast

$\prod_{\text {Catalytic site residues }}^{\text {Legend: }}$
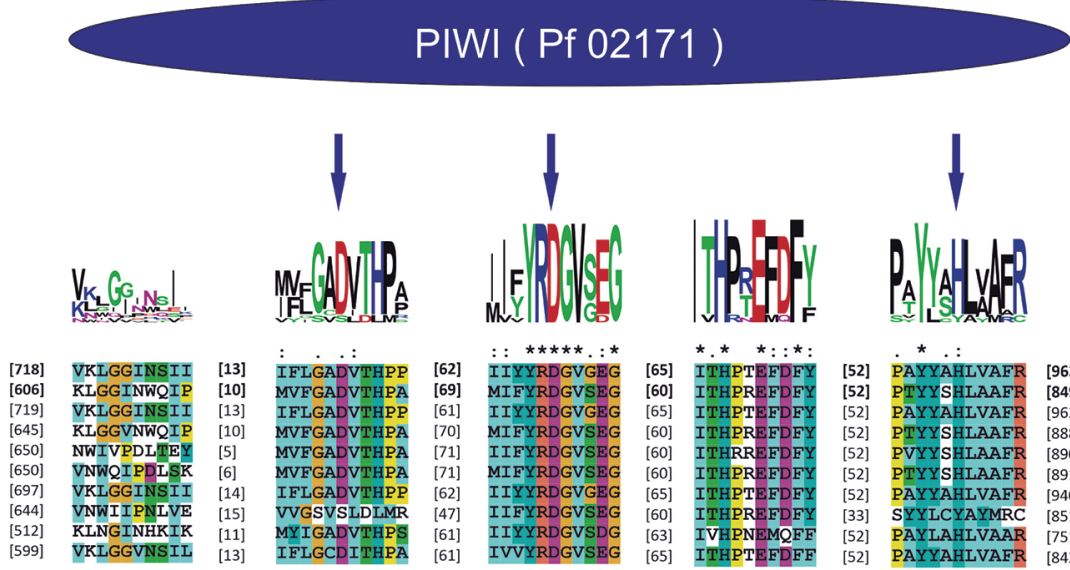

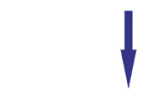

$\downarrow \downarrow$
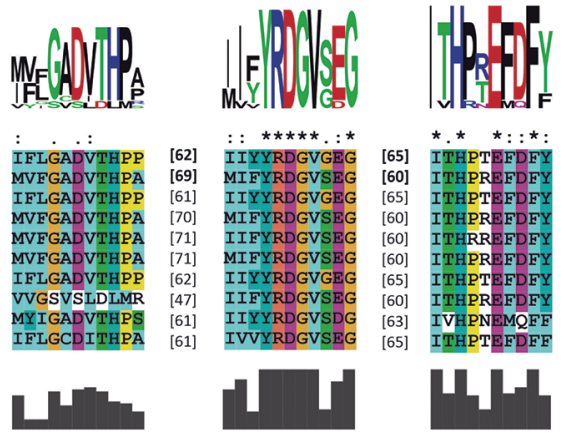

IFLGADVTHPP
MVFGADVTHPA

MVFGADVTHPA

MVFGADVTHPA
IFLGADVTHPP

MYGSVADLTHPS

MYIGADVTHP
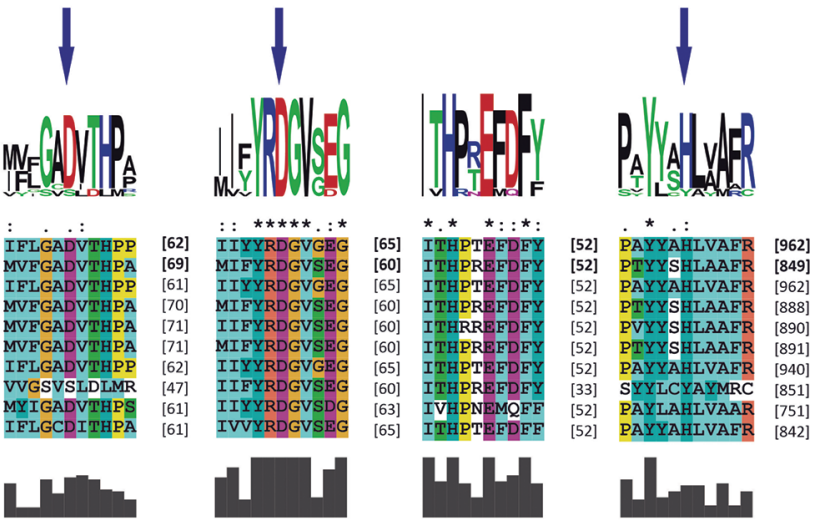

Fig. 2: analysis of PIWI conserved domain of Schistosoma haematobium AGO proteins.

two miRNAs genes to consider them clustered), such as sht-miR-71a/sht-miR-2a-1/sht-miR-2b/sht-miR-2e [Supplementary data I (Fig. 2)].

All pre-miRNAs identified were analysed for their structural and thermodynamic characteristics. The $S$. haematobium Egypt pre-miRNAs displayed MFE with an average of $-25.3 \mathrm{kcal} / \mathrm{mol}$, with values between -48.5 and $-18.5 \mathrm{kcal} / \mathrm{mol}$; AMFE values averaging $-27.91 \mathrm{kcal} /$ mol and MFEI with $-0.752 \mathrm{kcal} / \mathrm{mol}$ [Supplementary data II (Table III)].

In addition, we performed statistical analyses using thermodynamic and structural characteristics of premiRNAs found in $S$. haematobium Egypt compared to pre-miRNAs deposited in the miRBase from Ecdysozoa, Cnidaria, Polifera and Schistosoma clades [Supplementary data II (Table IV)]. We compared the miRNA precursor characteristics from genus Schistosoma miRNA precursors and no differences were observed among the characteristics analysed ( $\mathrm{p}>0.05)$. On the other hand, in the analysis performed among $S$. haematobium Egypt against Ecdysozoa, Cnidaria and Porifera phylum, the pre-miRNA characteristics were significantly different ( $p$ $<0.05)$.

Results obtained using SRA data from male (SRR6655496) and female worms (SRR6655494) evidenced that all mature miRNAs were found in the genome, although some were not expressed in the libraries used for analysis. On the other hand, highly conserved miRNAs such as bantam, miR-2, let-7, miR-71, miR125, miR-124 and miR-10 showed a greater amount of reads found in SRA data. In addition, some miRNAs were found only in the adult males library, suggesting possible targets for further studies [Supplementary data II (Table V)].

Of all precursor miRNAs, four families (miR10, miR-8, miR-2 and miR-7) were selected to further analysis once they showed high conservation based on structural, thermodynamic characteristics and their phylogenetic distribution.
sht-miR-10 - The sht-miR-10 precursor found in this study, showed two mature miRNAs, sht-miR-10-3p and sht-miR-10-5p. The sht-miR-10 precursor sequence presented a great conservation in the primary and secondary structures when compared to the ortholog sequences used for these analyses, as S. mansoni, S. japonicum, Apis mellifera and D. melanogaster (Figs 7, 8).

Three clades were observed in the tree generated by phylogenetic analysis. The first clade was identified as Lophotrochozoa, showing sequences of Mollusca and Platyhelminthes organisms. The second clade, called Ecdysozoa, was composed by arthropods organism sequences and the third clade presented Echinodermata organisms. This distribution showed similarity to the tree of life, where $S$. haematobium Egypt remained alongside others flatworms (Fig. 9).

sht-miR-8 - One precursor of miR-8 family was identified in the $S$. haematobium Egypt genome, sht-miR-8. Two mature miRNAs from this precursor also were found and named sht-miR-8-3p and sht-miR-8-5p. These sequences presented high conservation in relation to their orthologs in S. mansoni, S. japonicum, Bombix mori, Heliconius melpomene, Anopheles gambiae, Daphnia pulex, Nasonia vitripennis, Ixodes scapularis and Lottia gigantea, both for analyses of primary structure and secondary structures [Supplementary data I (Figs 3, 4)].

The phylogenetic analysis generated a tree divided in two clades: Lophotrochozoa and Ecdysozoa. The $S$. haematobium Egypt sequences were found closer to the main orthologs species, S. japonicum and S. mansoni, in the clade Lophotrochozoa, as well as organisms belonging to the Mollusca phylum. For the Escysozoa clade, the arthropod organisms were the only representative of this group. According to the conservation of the pre-miRNA sequences of these species, this distribution relation is also observed in the animal's tree of life [Supplementary data I (Fig. 5)]. 


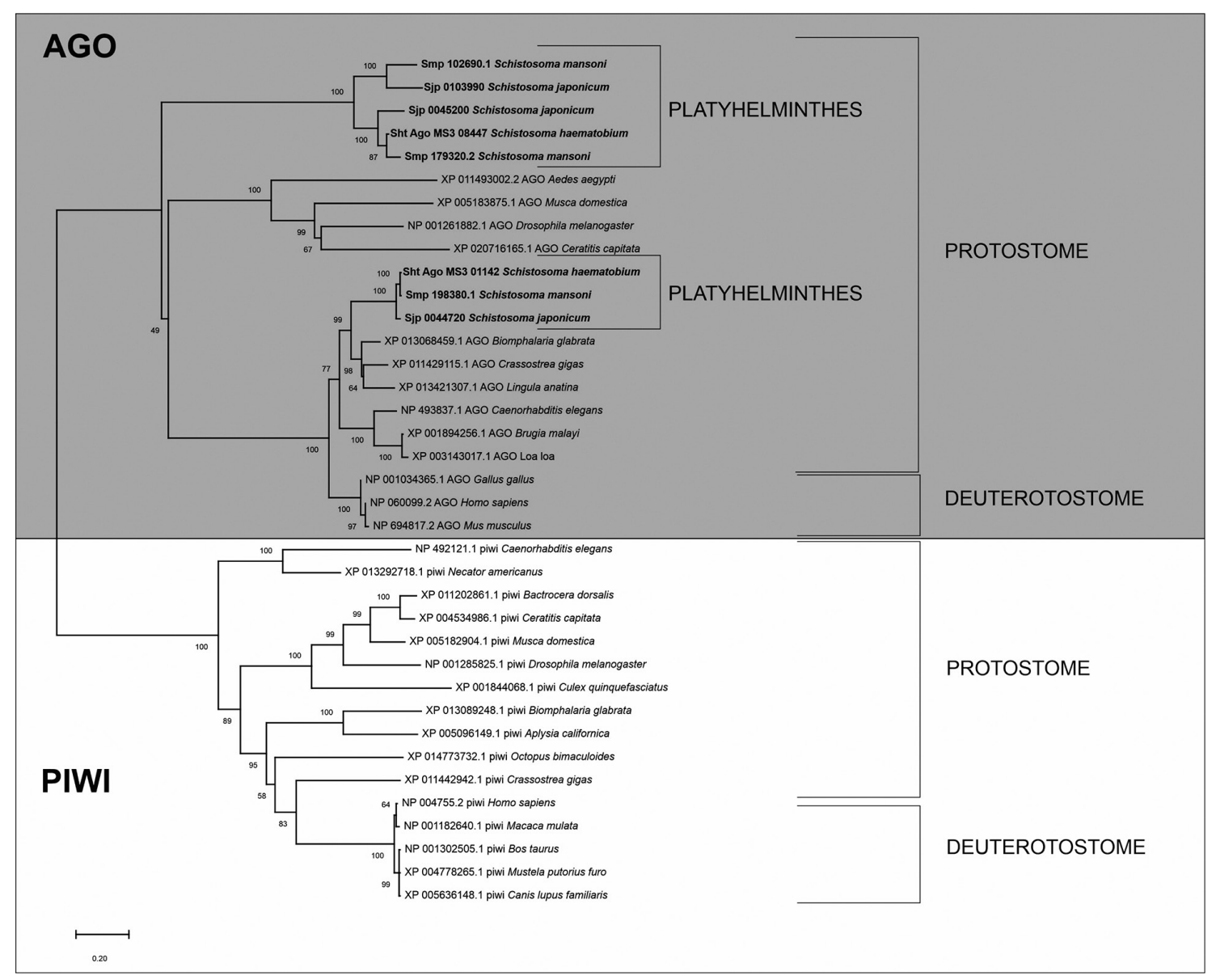

Fig. 3: phylogenetic analysis of Schistosoma haematobium AGO proteins and their orthologous.

sht-miR-2 - In miR-2 family of S. haematobium Egypt were identified five precursor miRNAs (sht-miR-2a-1, shtmiR-2a-2, sht-miR-2b, sht-miR-2c and sht-miR-2e) and nine mature miRNAs: sht-miR-2a-1-3p, sht-miR-2a-1-5p, sht-miR-2a-2-3p, sht-miR-2b-3p, sht-miR-2b-5p, sht-miR2c-3p, sht-miR-2c-5p, sht-miR-2e-3p and sht-miR-2e-5p.

The $S$. haematobium Egypt miRNAs from this family exhibited high conservation as mature miRNAs when compared with their orthologs in S. mansoni and S. japonicum [Supplementary data I (Fig. 6)]. It was possible to observe that there is a great similarity between the secondary structures of the pre-miRNAs within the same family, as well as between $S$. haematobium and $S$. mansoni [Supplementary data II (Fig. 7)].

The tree generated from the phylogenetic analysis of miR-2 family showed a distribution into two clades. The clade Lophotrochozoa was composed by the Mollusca, Annelida and Platyhelminthe phylum, while the Ecdysozoa clade presented Arthropod organisms as its representative. Besides that, there was a correct grouping between all precursors sequences of this family (sht-miR-2a-1, shtmiR-2a-2, sht-miR-2b, sht-miR-2c and sht-miR-2e) with their respective orthologs species, mainly $S$. mansoni and S. japonicum [Supplementary data I (Fig. 8)].

sht-miR-7 - Two precursor miRNAs of miR-7 family were identified: sht-miR-7 and sht-miR-7b. Besides that, three mature miRNAs also were found (sht-miR-7-5p and sht-miR-7b-5p) in S. haematobium Egypt genome. In the primary structure results of miR-7 family were observed a high conservation between the nucleotide sequences of $S$. haematobium miRNAs and their orthologs [Supplementary data I (Fig. 9)]. The analysis performed for pre-miRNA secondary structures of miR-7 family also evidenced a great conservation between the $S$. haematobium miRNAs and their orthologous organisms [Supplementary data I (Fig. 10)].

Three well defined clades were observed in the phylogenetic analysis performed for sht-miR-7 family: the Deuterostome group and the Lophotrochozoa and Ecdysozoa clades formed the Protostome group. The first group was composed of Chordata and Hemichordata phylum, while the second group had the presence of flatworms and arthropod organisms. The species classification showed by this analysis corroborates with the distribution found in the animal tree of life [Supplementary data I (Fig. 11)].

\section{DISCUSSION}

The first version of $S$. haematobium genome published by Young et al. provided the conditions to researchers to study the genome and we were able to apply this robust analysis to identify and characterise miRNAs, and the putative proteins involved in miRNA processing pathway in the genome of this human parasite. ${ }^{(16)}$ The 


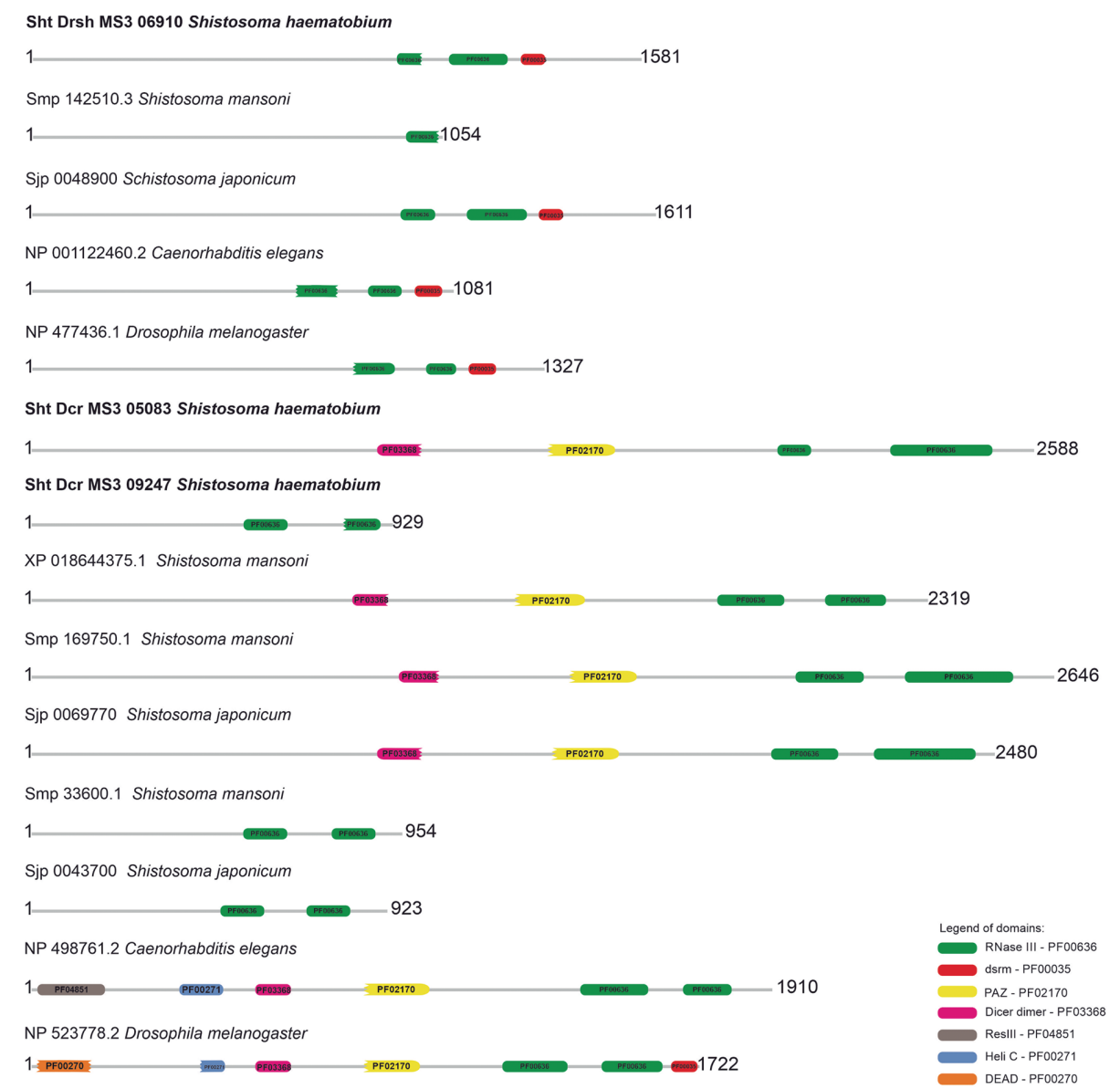

Fig. 4: conserved domains of Schistosoma haematobium DICER/DROSHA proteins and their orthologous from S. mansoni and S. japonicum, and model organisms Caenorhabditis elegans and Drosophila melanogaster.

miRNAs and proteins of their biosynthetic pathway were described in several organisms, including plants and animals. In animals, in the model organisms D. melanogaster and C. elegans, were found mature and precursor miRNAs, pathway proteins and miRNA target genes. ${ }^{(29,30)}$ In addition, in species from Schistosoma genus, such as $S$. mansoni and $S$. japonicum, also were identified these regulatory molecules, ${ }^{(10,31,32)}$ showing that there have been an evolutionary conservation of these molecules.

Stroehlein et al. ${ }^{(32)}$ described mature and precursor miRNAs in S. haematobium genome data, however, they did not mention the identification, characterisation and expression of the miRNA pathway genes in the organism under study, as observed in our results. In addition, we were able to identify conserved and novel miRNAs, being 149 mature miRNAs and 131 miRNA precursors. Stroehlein and colleagues found 36 conserved miRNAs and 53 new miRNAs, while we identified 41 conserved miRNAs in the Schistosoma genus and 108 conserved miRNAs in others animal species. Of all mature miRNAs identified in both studies, only 25 mature miRNAs were found in both studies. 124 novel conserved mature miRNAs were found in this study comparing to Stroehlein and colleagues work. We also have validated the presence of the mature $S$. haematobium miRNAs us- ing public smallRNA-seq libraries of male and female $S$. haematobium parasites. ${ }^{(32)}$

In miRNA processing and maturation, the proteins ARGONAUTE, DICER and DROSHA play a several key roles. In the nucleus, DROSHA complex protein is responsible for the first step in this pathway. The primiRNA is the product of the transcription characterised mainly by the formation of a stem-loop. This structure is targeted by DROSHA complex, that acting by the cleavage generate the pre-miRNAs. ${ }^{(33)}$ Protein DICER cleaves the pre-miRNAs removing the loop from the structure forming a miRNA duplex. In the RISC complex, DICER protein guides the miRNA duplex to ARGONAUTE proteins which one of the mature miRNA in the duplex target the target gene. A lot of others proteins are part of the RISC complex such as Fmr1, Tsn, VIG, among others. ${ }^{(34)}$

The family of ARGONAUTE proteins has two important subfamilies, AGO and PIWI. Both subfamilies play important roles in noncoding RNA pathways, being the AGO proteins the main protein of the RISC complex in the microRNA and small interfering RNA (siRNA) pathways, while PIWI proteins is responsible for specific RISC complex in PIWI-interecting RNAs (piRNA) pathway. ${ }^{(35)}$ This both ARGONAUTE subfamilies have shown two highly conserved domains, PIWI and PAZ 
$\left.\begin{array}{r}4- \\ 3- \\ \frac{2}{2} 2- \\ 1- \\ 0\end{array}\right]$

Sht Drsh MS3 06910 Schistosoma haematobium Smp $142510.3 \bar{S}$ chistosoma mansoni Sjp 0048900 Schistosoma japonicum NP 477436.1 Drosophila melanogaster NP-001122460.2 Caenorhabditis elegans Sht Dcr MS3 05083 Schistosoma haematobium Sht Der MS3 09247 Schistosoma haematobium XP_018644375.1 Schistosoma mansoni Smp 169750 Schistosoma mansoni Sjp 0069770 Schistosoma japonicum Sjp 0043700 Schistosoma japonicum Smp 033600.1 Schistosoma mansoni NP 498761.2 Caenorhabditis elegans NP 523778.2 Drosophila melanogaster

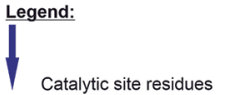

RiboC (Pf00636)

It

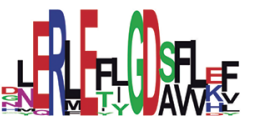

$: *: * \quad * *: .:$.

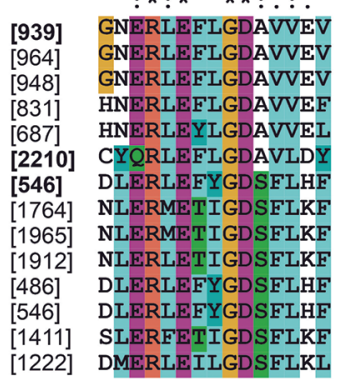

[1222] DMERLEILGDSFLKL

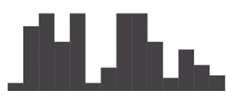

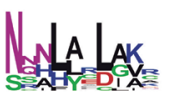

$\begin{array}{lll}{[27]} & \text { NQHLAELAVR } & {[21]} \\ {[27]} & \text { NQHLAELAVR } & {[21]} \\ {[27]} & \text { NQHLAELAVR } & {[21]} \\ {[27]} & \text { NQHLALLAKK } & {[21]} \\ {[27]} & \text { NRNLATLAKN } & {[21]} \\ {[27]} & \text { NNIFAALAVR } & {[13]} \\ {[27]} & \text { SNAHLCDIAC } & {[42]} \\ {[27]} & \text { NSNLYRLGKA } & {[107]} \\ {[27]} & \text { NSNLYRLGKA } & {[107]} \\ {[27]} & \text { NSNLYRLGKT } & \\ {[27]} & \text { SNAHLCDIAC } & {[42]} \\ {[27]} & \text { SNAHLCDIAC } & {[42]} \\ {[27]} & \text { NCNLYRLGKR } & {[107]} \\ {[27]} & \text { NRNLLFCLID } & {[103]}\end{array}$

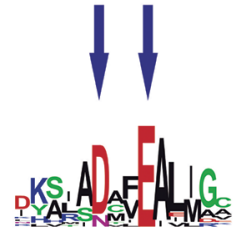

IYARSDAFEATMDE [1025 IYARSDAFEALMGK [1050] IYARSDAFEAIMAA [1034] RHAMANCFEALMGA [917] KHALANAFEAVMAA [773] LLHTIDVFEIEIRQ [2288] DTSIADI DKSLADI ALIGC [653] DKSIADCVEALIGC [1937] DKSIADCVEALIGC [2138] -II-_ [1927] DKSLADMVA I IGC [593] DKST ADMVAT [653] DKSLADMVEAIIGC [653] DKSIADAVEALIGV [1583] NKVIADTLEALLGV [1376]

Fig. 5: analysis of ribonuclease III (Riboc I and II) conserved domains of Schistosoma haematobium DICER and S. haematobium DROSHA proteins and their orthologous.

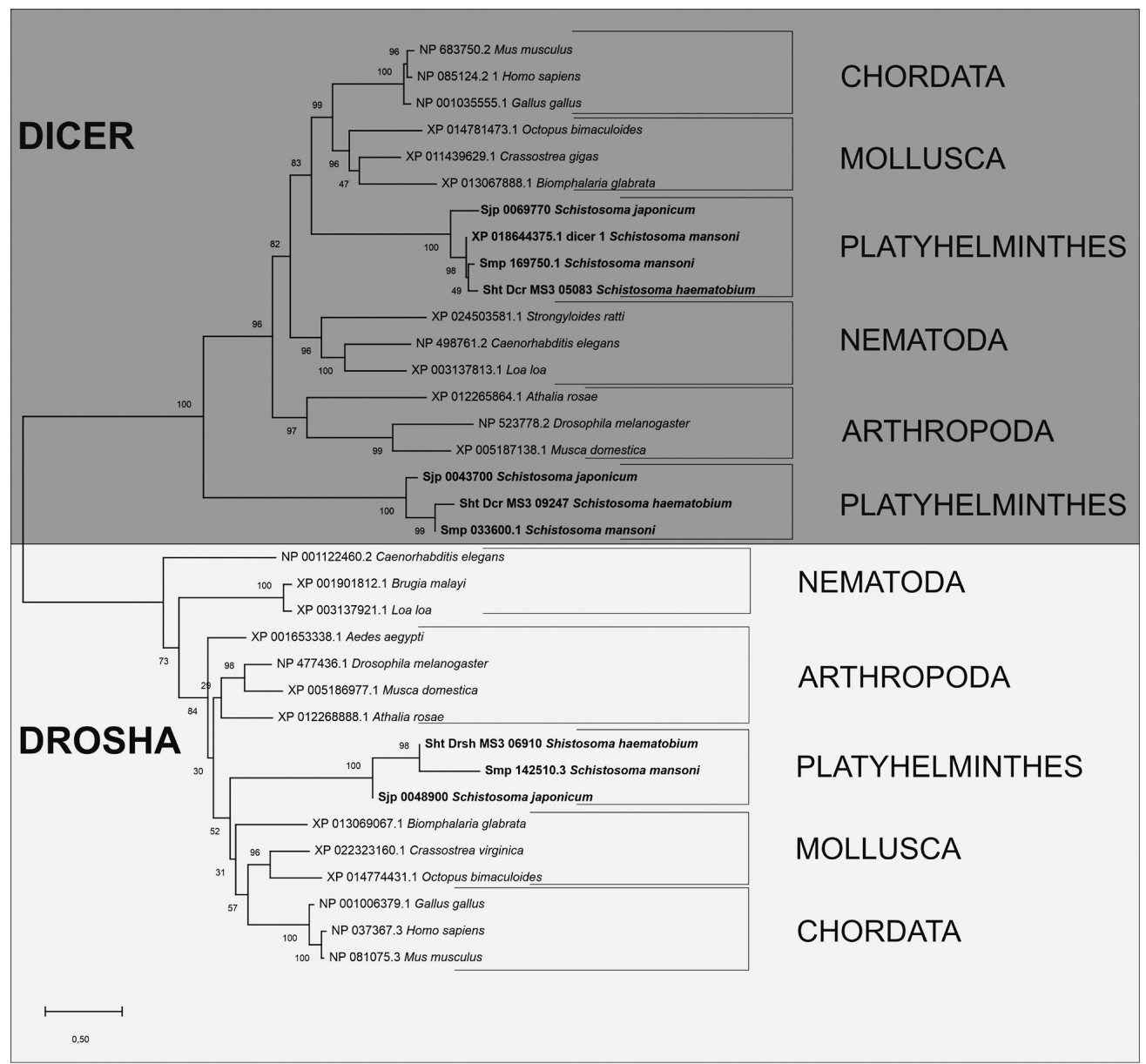

Fig. 6: phylogenetic analysis of the Schistosoma haematobium DICER and S. haematobium DROSHA proteins and their orthologous. 


\section{sht-miR-10}
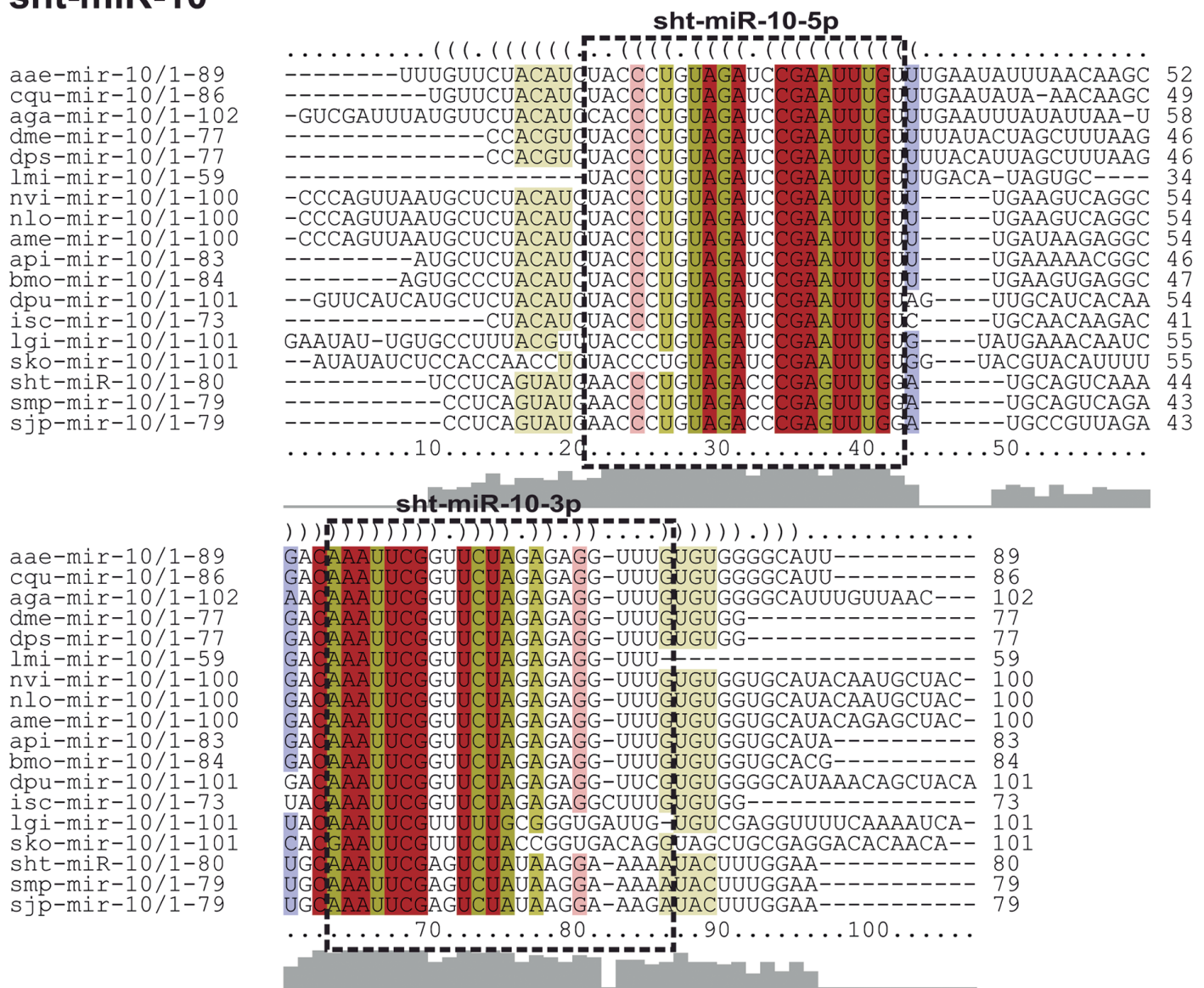

Fig. 7: alignment of the sht-miR-10 pre-miRNA and their orthologs; sht: Schistosoma haematobium; smp: S. mansoni; sjp: S. japonicum; sko: S. kowalevskii; lgi: Lottia gigantea; isc: Ixodes scapularis; dpu: Daphnia pulex; bmo: Bombyx mori; api: Acyrthosiphon pisum; ame: Apis mellifera; nvi: Nasonia vitripennis; nlo: Niphona longicornis; lmi: Locusta migratoria; dps: Drosophila pseudoobscura; dme: D. melanogaster; aga: Anopheles gambiae; cqu: Culex quinquefasciatus; and aae: Aedes aegypti.

domains. The PAZ domain has been presented at the Nterminal of the protein, while the $\mathrm{C}$-terminal region has been composed by PIWI domain. The positions of theses domains are strategic, since they have the ability to position the small RNA in its target gene. ${ }^{(36,37)}$ The PIWI domain, in particular, has a catalytic site responsible for cleaving target messenger RNA (mRNA) of the miRNA. This active site has been composed by three amino acid residues: DDH (aspartate, aspartate and histidine) or DDD (three aspartates). ${ }^{(36)}$

All of these aspects in our analysis had a great importance. The putative ARGONAUTE genes found in S. haematobium Egypt showed the conserved domains, PAZ and PIWI. The phylogenetic tree showed two distinct clades. One clade showed only AGO orthologue proteins including sht_Ago_MS3_08447 and sht_Ago_ MS3 0114 suggesting that these sequences are ARGONAUTE proteins. Furthermore, the catalytic site was identified in the PIWI domain of the S. haematobium Egypt ARGONAUTE proteins (DDH) corroborating with PIWI domain catalytic site reported by the literature. These proteins are considered one of the most important proteins involved in the miRNAs pathway, be- cause they are catalytic component of RISC complex and help in the high silencing capacity of the miRNAs. ${ }^{(38,39)}$

All conserved domains described for the DICER and DROSHA proteins were found in the analyses of this work, where DROSHA showed the same conserved domains reported by others studies and DICER presented, besides the mentioned domains for this protein, one Dicer Dimer domain. It is important to emphasise that this analysis was possible once we used the new gene prediction beyond the predicted proteome from SchistoDB. We were able to improve the prediction of putative protein $S$. haematobium DROSHA. The presence of these domains found in S. haematobium Egypt DICER and DROSHA confirm that these proteins are ortholog of DICER and DROSHA. The DICER protein contains three main conserved domains, one PAZ domain and two domains called RNase III. ${ }^{(15,39)}$ In $S$. mansoni, in the sequences identified as DROSHA, were found three conserved domains: two RNase III and one DSRM domain..$^{(15)}$

In addition, the analyses performed to identify the active site of two RNase III domains of DICER showed the distribution of following amino acids: glutamic acid (E), aspartate (D), aspartate (D) and glutamic acid (E), 

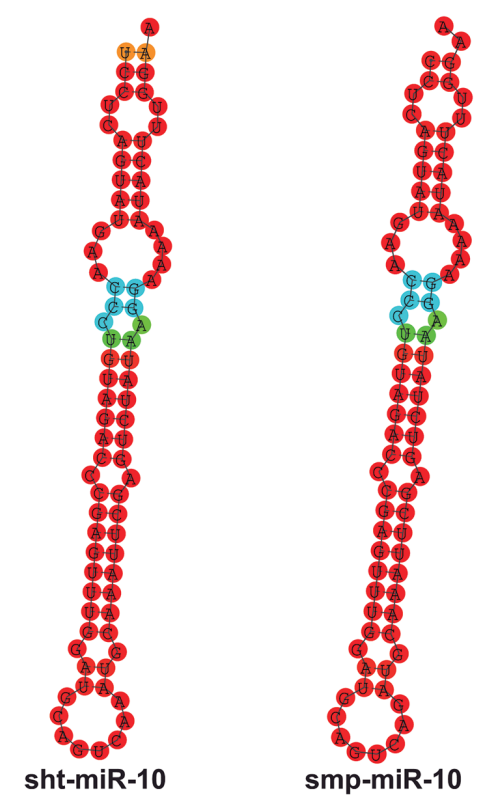

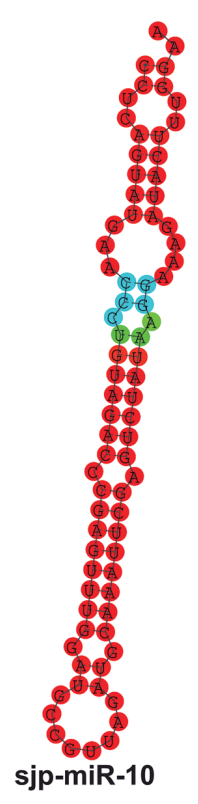

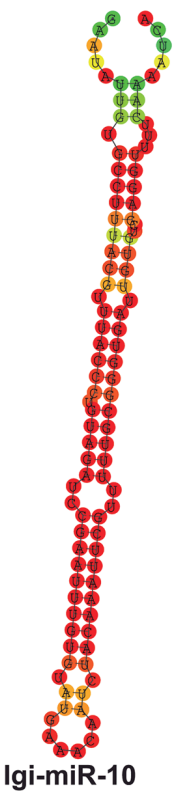

Igi-miR-10

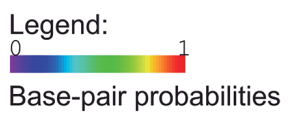

Fig. 8: secondary structures of the sht-miR-10 pre-miRNA and their orthologs; sht: Schistosoma haematobium; smp: S. mansoni; sjp: S. japonicum, dme: Drosophila melanogaster and lgi: Lottia gigantea.

forming the site EDDE also present in S. mansoni. ${ }^{(15)}$ These findings found in DICER, DROSHA and AGOlike proteins of $S$. haematobium corroborate with the literature in domain distribution, conservation of active site and phylogenetic distribution confirming Thais prediction within $S$. haematobium data.

In the expression analysis using RNAseq data SRR6655495, SRR6655497, SRR6655493 - the S. haematobium transcripts of ARGONAUTE, DROSHA and DICER showed in general low expression level in all three stages of development analysed. The highest expression way round for the VIG gene explained by the importance of this gene not only in the RISC complex of miRNA pathway but also in other mechanisms in the parasite such as the formation and maintenance of heterochromatin. ${ }^{(40)}$

24 mature miRNA sequences identified corroborated with Stroehlein et al. ${ }^{(32)}$ study such as bantam, let-7, miR124 and others. Some studies involving S. japonicum were performed to identify miRNAs based on computational and experimental analysis. Xue et al. ${ }^{(31)}$ described five novel miRNAs while Huang et al. found 176 mature miRNAs using computational analyses. ${ }^{(41)}$ In $S$. mansoni 67 mature miRNAs and 42 precursor miRNAs were identified by Gomes et al..$^{(10)}$ and 11 mature miRNAs were confirmed by experimental analyses by Simões et al. ${ }^{(42)}$ A comparison between $S$. mansoni and S. haematobium Egypt data showed that among the conserved miRNAs found in S. mansoni, only five of them were not identified in $S$. haematobium Egypt genome data in our study.

Most of the miRNA precursor genes was found in the intergenic regions of the S. haematobium Egypt genome [Supplementary data II (Table II)] corroborating with most animal species. We found 15 miRNAs in clusters. MiRNAs distributed in clusters has also been showed in other organisms such as in S. japonicum, S. mansoni and B. glabrata $^{(10,41,43,44,45)}$ The miRNA cluster gene mir$71 / 2 a-1 / 2 b / 2 e$ found in this work [Supplementary data I (Fig. 2)] has been reported in other organisms such as $S$. mansoni. ${ }^{(10)}$ The miRNA antiparallel clusters had been also occasionally found in other animals genomes deposited in miRBase version 22 (http://www.mirbase. org/), such as smp-miR-124b and smp-miR-124a from S. mansoni, mmu-miR-124-2 and mmu-miR-124b from Mus musculus, and oni-miR-124a-3 and oni-miR-124b-1 from Oreochromis niloticus, corroborating to our results [Supplementary data II (Table II)].

The pre-miRNAs sequences of $S$. haematobium Egypt displayed a Minimal Energy Free (MFE) with a mean of $-25.3 \mathrm{kcal} / \mathrm{mol}$, with values between -48.5 and $-18.5 \mathrm{kcal} / \mathrm{mol}$, and, considering the structural and thermodynamic characteristics already reported for miRNAs, a precursor miRNA molecule is stable and can generate mature miRNA having a value of the MFE of $-20 \mathrm{kcal} / \mathrm{mol}$. Besides that, this data corroborating with the values found in others animals studies, beyond corresponding to the values used for the orthologs $S$. mansoni and $S$. japonicum. ${ }^{(10,42)}$ The pre-miRNA size $(\sim 70 \mathrm{nt})$ of S. haematobium miRNAs corroborated to orthologous size, indicate a high probability that these sequences are real pre-miRNA sequences.

In addition, the guanine-cytosine (GC) content found in the results of this work showed a distribution between $21.28 \%$ and $45.94 \%$ of GC content, but the majority of the sequences presented a percentage greater than $25 \%$. This 


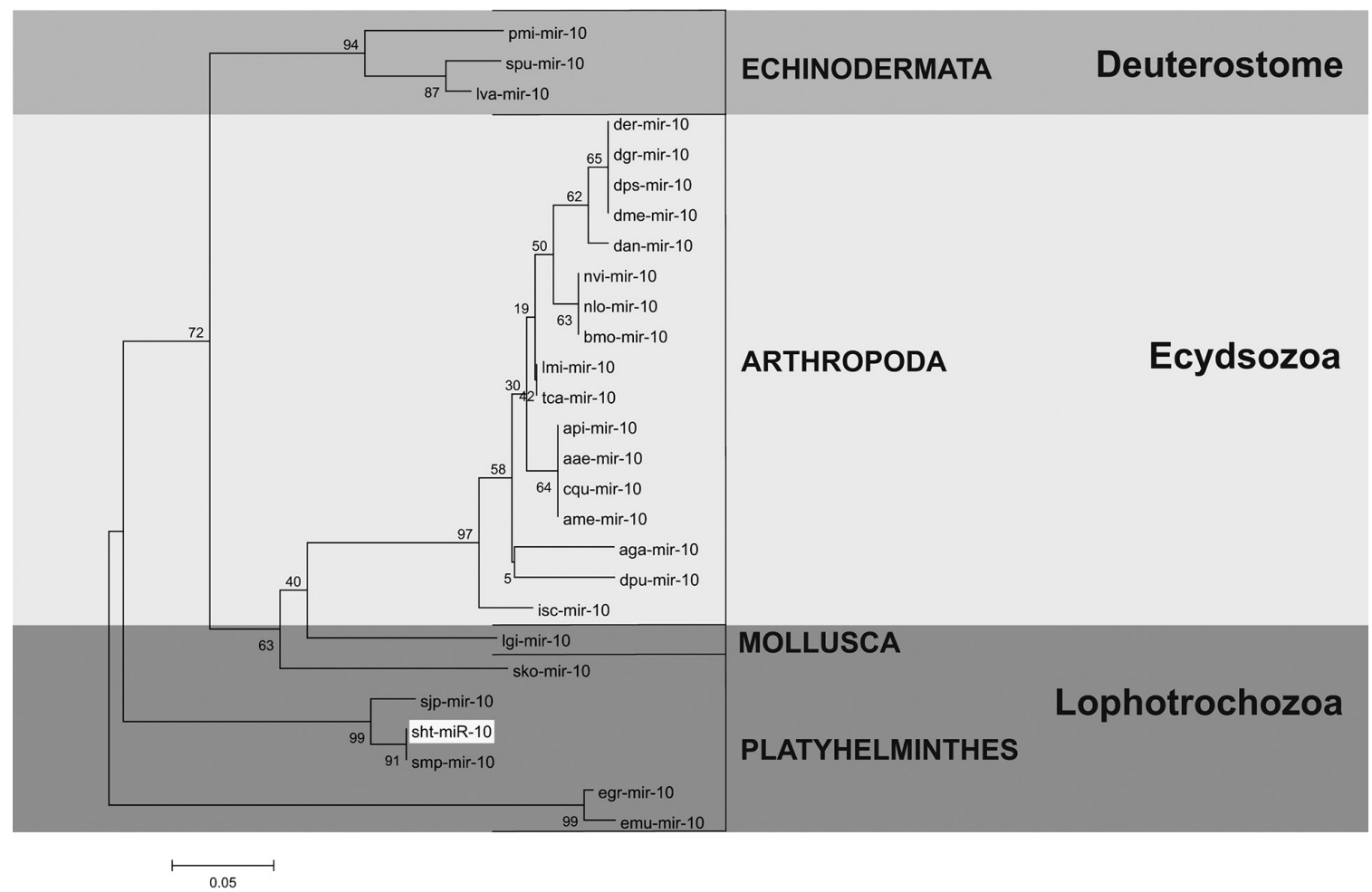

Fig. 9: phylogenetic tree performed to sht-miR-10 pre-miRNA identified in Schistosoma haematobium Egypt genome and their orthologs; sht: S. haematobium; smp: S. mansoni; sjp: S. japonicum; egr: Echinococcus granulosus; emu: Echinococcus multilocularis; lgi: Lottia gigantean; isc: Ixodes scapularis; dpu: Daphnia pulex; aga: Anopheles gambiae; ame: Apis mellifera; cqu: Culex quinquefasciatus; aae: Aedes aegypti; api: Acyrthosiphon pisum; tca: Tribolium castaneum; lmi: Locusta migratoria; bmo: Bombyx mori; nvi: Nasonia vitripennis; nlo: Niphona longicornis; dan: Drosophila ananassae; dme: Drosophila melanogaster; dgr: D. grimshtwi; dps: D. pseudoobscura; der: D. erecta; lva: Lytechinus variegatus; spu: Strongylocentrotus purpuratus; and pmi: Patiria miniata.

is one of the main parameters for pre-miRNAs identification and the presence of these nucleotides is very important for the stability of pre-miRNA secondary structure. In animals, evaluation value of this parameter should be found between $20 \%$ and $55 \%$. As showed, the results indicated that the sequences analysed satisfy the requirements to be considered miRNA precursors. Previous works have shown how important has been the use of these structural and thermodynamic characteristics for conserved miRNA elucidation and prediction in the genome. ${ }^{(10)}$

The statistical analyses using thermodynamic and structural characteristics of the S. haematobium Egypt pre-miRNAs, when comparing the genus Schistosoma showed no differences between the groups $(p>0.05)$. Both displayed high conservation and similarity among pre-miRNAs from S. haematobium Egypt and species belonging to the genus Schistosoma. However, the results for S. haematobium Egypt were significantly different from results for other clades such as Ecdysozoa, Cnidaria and Porifera $(p<0.05)$, suggesting an evolutionary conservation of the pre-miRNA characteristics within each animals clade (superphylum, phylum or genus) and a significant difference among different clades [Supplementary data II (Table IV)]. The studies based on specific characteristics of mature and precursor miRNAs have been used in sequence groups in different species, from Protista to Metazoa and Plantae..$^{(11,46,47,48)}$
Our analyses using the libraries of small RNAs from male and female adult worms showed a validation of all 149 mature miRNAs found in the genome of S. haematobium Egypt [Supplementary data II (Table V)]. Some conserved miRNAs such as bantam-3p, let-7-3p, miR124-3p and miR-10-5p demonstrated a large number of reads in two libraries studied, corroborating with the results found in a previously published study, ${ }^{(32)}$ however, our findings refer to mature miRNAs. Other miRNAs have also identified in both studies, such as the miRNAs of the miR-2, miR-7 and miR-8 families demonstrating the importance of these molecules in both libraries, female and male adult worms. ${ }^{(32)}$

The miR-10 was found in S. haematobium Egypt genome containing two mature miRNAs (sht-miR-10-5p and sht-miR-10-3p), corroborating with results observed in studies with $D$. melanogaster and $S$. japonicum. ${ }^{(43,49)}$ Besides that, when compared to the pre-miR-10 sequences of $S$. mansoni, S. haematobium Egypt and $S$. japonicum had $100 \%$ identity in the region of their two mature miRNAs (Fig. 7). Many different animal species have shown this miRNA and its presence in the HOX cluster, composed by miR-10 and HOX genes. This relationship was observed in organisms such as S. mansoni, A. gambiae, Tribolium castaneum, zebrafish and human. ${ }^{(10,50)}$ On the other hand, miR-10 was one of five miRNAs considered pathogen-specific found in rabbits infected 
with $S$. japonicum, which may offer a new biomarker to diagnose schistosomiasis. ${ }^{(51)}$

The results miR- 8 precursor shown two mature miRNAs. Besides that, in S. haematobium a high conservation was observed among the species belonging to Lophotrochozoa and Ecdysozoa clades, being considered a Protostome-specific miRNA, corroborating with $S$. mansoni data. ${ }^{(52)}$ This miRNA was found in several organisms, including the model organism Drosophila sp. that was also identified by computational analyses. ${ }^{(49)}$ In another study, miR-8 was identified in two mosquito's vector: Aedes albopictus and Culex quinquefasciatus. ${ }^{(53)}$ In S. japonicum, miR-8 also was identified and presented a greater expression in mature adult worms, but was observed with some nucleotide substitutions considered specific for Schistosoma sp. ${ }^{(43)} \mathrm{MiR}-8-3 \mathrm{p}$ was found 11 times more reads in the male library compared to the amount of reads found in the female library which were 104 reads. For mature miRNA miR-8-5p, none read was found in the male library and in the female library.

The miR-2 family showed five precursors and ten mature miRNA in $S$. haematobium data. This family is specific of protostome organisms, since in the phylogenetic tree were found only organisms from Ecdysozoa and Lophotrochozoa clades, corroborating with the results shown by Gomes et al. ${ }^{(52)}$. Besides that, miR-2 family has considered one of the larger miRNA family found in D. melanogaster presenting eight miRNAs in the genome. ${ }^{(54)}$ On the other hand, in C. elegans, another model organism, there is only one sequence of this family. ${ }^{(55)}$ In $S$. mansoni, nine members belonging to the miR-2 family were identified and some were in the cluster with miR-71.(10) This cluster is located on a sexual chromosome in the parasite and therefore, may play a role in their sexual maturation. ${ }^{(44)}$

The sequences of miR-7 family corroborated with miRNAs found in S. mansoni. In addition, the secondary structures performed for miR-7b, in both studies, show a high conservation of these sequences. ${ }^{(52)}$ This family is one of the most conserved miRNA families and was found in all Bilateria, including flatworms, nematodes, insects and vertebrates. ${ }^{(56)}$ In $S$. japonicum, miR-7 was more expressed in male worms than in females; on the other hand, this miRNA is expressed in a specific stage of parasite life, mainly in cercariae stage. Data involving the validation of these miRNAs showed that in the small RNA library of adult male there were more reads of miR7 compared to adult female library. The mature miRNA miR-7-5p demonstrates 25 times more reads in males library compared to females library. The miR-7 is related with different cellular processes. In Drosophila sp., it was reported its involvement in the formation of photoreceptor organs ${ }^{(57)}$ and in a study with rat pancreas was observed that miR-7 is the miRNA most abundant in this cell type, confirming the performance flexibility of this miRNA. ${ }^{(58)}$

According to the results obtained in this work, through bioinformatics and computational analyses we were able to identify 14 key proteins involved in small RNA processing pathway. Our results showed that the miRNA pathway genes were transcriptionally active. In addition, we identified 149 mature miRNAs and 131
pre-miRNAs in the genome of this species, being highly conserved in their sequences, corroborating with others studies involving $S$. mansoni and $S$. japonicum. The results found in this work will open up a new avenue for studying the miRNAs in the Schistosome biology and technologies involving the gene silencing control in this organism. In addition, will allow understanding many processes and mechanisms in their biology, since these molecules present a significant regulatory role for different mRNAs, affecting various characteristics of the organism in different stages and environmental conditions.

\section{ACKNOWLEDGEMENTS}

To the members of the Laboratory of Bioinformatics and Molecular Analysis (LBAM - UFU) for providing computational structure and for helping with the data mining and the figure/table organisation.

\section{AUTHORS' CONTRIBUTION}

MSG, TCSC, CBA, LGP and LGAM - Proposed the research; MSG, TCSC, CBA, LGP and LGAM designed the experiments; TCSC, CBA, LGP, LGAM, TCA and LRA - prepared samples; TCSC, CBA, LGP, TCA, GCS, THCR, PEG and LRA - performed experiments; TCSC, CBA, LGP and ERM - wrote manuscripts and prepared figures; MSG - supervised the project. All authors reviewed the manuscript. The authors declare that there is no conflict of interest, whether financial or non-financial origin, involved in the publication of this article. The data present in this article have not been published and are not under consideration elsewhere, moreover we approved the submission of the manuscript.

\section{REFERENCES}

1. Mohammed K, Abdullah MR, Ikeh EI, Isamail A, Omar J, Popoola FJ. A multivariate analysis on the assessment of risk factors associated with infections and transmission of schistosomiasis haematobium in some selected areas of North-Western, Nigeria. J Med Bioeng. 2015; 4(1): 7-11.

2. McManus DP, Dunne DW, Sacko M, Utzinger J, Vennervald BJ, Zhou XN. Schistosomiasis. Nat Rev Dis Primers. 2018; 4(13): 1-19.

3. Risikat SA, Ayoade AA. Correlation analysis between the prevalence of Schistosoma haematobium and water conditions: a case study among the school pupils in southwestern Nigeria. IJRRAS. 2012: 13(1): $160-5$.

4. Stephenson L. The impact of schistosomiasis on human nutrition. Parasitology. 1993; 107(1): 107-23.

5. Richards CS. Genetics of a molluscan vector of schistosomiasis. Nature. 1970; 227(5260): 806-10.

6. Lewis FA, Richards CS, Knight M, Cooper LA, Clark B. Schistosoma mansoni: analysis of an unusual infection phenotype in the intermediate host snail Biomphalaria glabrata. Ex Parasitol. 1993; 77(3): 349-61.

7. Bartel DP. MicroRNA target recognition and regulatory functions. Cell. 2009; 136(2): 215-33.

8. Moazed D. Small RNAs in transcriptional gene silencing and genome defence. Nature. 2009; 457: 413-20.

9. Winter J, Jung S, Keller S, Gregory RI, Diederichs S. Many roads to maturity: microRNA biogenesis pathways and their regulation. Nat Cell Biol. 2009; 11(3): 228-34.

10. Gomes MS, Muniyappa MK, Carvalho SG, Guerra-Sá R, Spillane 
C. Genome-wide identification of novel microRNAs and their target genes in the human parasite Schistosoma mansoni. Genomics. 2011; 98(2): 96-111.

11. Li L, Xu J, Yang D, Tan X, Wang H. Computational approaches for microRNA studies: a review. Mamm Genome. 2010; 21: 1-12.

12. Chang SH, Tang P, Lai CH, Kuo ML, Wang LC. Identification and characterisation of microRNAs in young adults of Angiostrongylus cantonensis via a deep-sequencing approach. Mem Inst Oswaldo Cruz. 2013; 108(6): 699-706.

13. Ambros V. MicroRNA pathways in flies and worms: growth, death, fat, stress, and timing. Cell. 2003; 113(6): 673-6.

14. Lee YS, Nakahara K, Pham JW, Kim K, He Z, Sontheimer EJ, et al. Distinct roles for Drosophila Dicer-1 and Dicer-2 in the siRNA/ miRNA silencing pathways. Cell. 2004; 117(1): 69-81.

15. Gomes MS, Cabral FJ, Jannotti-Passos LK, Carvalho O, Rodrigues V, Baba EH, et al. Preliminary analysis of miRNA pathway in Schistosoma mansoni. Parasitol Int. 2009; 58 (1): 61-8.

16. Young ND, Jex AR, Li B, Liu S, Yang L, Xiong Z, et al. Wholegenome sequence of Schistosoma haematobium. Nat Genet. 2012; 44(2): 221-5.

17. Dobin A, Davis CA, Schlesinger F, Drenkow J, Zaleski C, Jha S, et al. STAR: ultrafast universal RNA-seq aligner. Bioinformatics. 2013; 29(1): 15-21.

18. Mitrophanov AY, Lomsadze A, Borodovsky M. Sensitivity of hidden Markov models. J Appl Probab. 2005; 42(3): 632-42.

19. Kent WJ. BLAT: the blast-like alignment tool. Genome Res. 2002; 12(4): 656-64.

20. Mall T, Eckstein J, Norris D, Vora H, Freese NH, Loraine AE. ProtAnnot: an App for integrated genome browser to display how alternative splicing and transcription affect proteins. Bioinformatics. 2016; 32(16): 2499-2501.

21. Rice P, Longden I, Bleasby A. EMBOSS: the European molecular biology open software suite. Trends Genet. 2000; 16(6): 276-7.

22. Lorenz R, Bernhart SH, Siederdissen CHZ, Tafer H, Flamm C, et al. ViennaRNA Package 2.0. Algorithms Mol Biol. 2011; 24: 6-26.

23. Kroll KW, Mokaram NE, Pelletier AR, Frankhouser DE, Westphal MS, Stump PA, et al. Quality control for RNA-Seq (QuaCRS): an integrated quality control pipeline. Cancer Inform. 2014; 13(3): 7-14.

24. Bolger AM, Lohse M, Usadel B. Trimmomatic: a flexible trimmer for Illumina sequence data. Bioinformatics. 2014; 30(15): 2114-20.

25. Friedländer MR, Mackowiak SD, Li N, Chen W, Rajewsky N. MiRDeep2 identificam com a contagem de novos genes de microRNA em sete clados de animais. Nucleic Acids Res. 2012; 40(1): 37-52.

26. Tamura K, Peterson D, Peterson N, Stecher G, Nei M, Kumar S. MEGA5: molecular evolutionary genetics analysis using maximum likelihood, evolutionary distance, and maximum parsimony methods. Mol Biol Evol. 2011; 28(10): 2731-9.

27. Saitou N, Nei M. The neighbor-joining method: a new method for reconstructing phylogenetic trees. Mol Biol Evol. 1987; 4(4): 406-25.

28. Hair Jr JF, Black CW, Babin BJ, Anderson RE. Multivariate data analysis. 7th ed. Prentice Hall; 2009.

29. Vella MC, Slack FJ. C. elegans microRNAs. In: WormBook: the online review of $C$. elegans Biology [Internet]. Pasadena: WormBook; 2005. Available from: https://www.ncbi.nlm.nih.gov/ books/NBK19796/
30. Carthew RW, Agbu P, Giri R. MicroRNA function in Drosophila melanogaster. Semin Cell Dev Biol. 2017; 65: 29-37.

31. Xue X, Sun J, Zhang Q, Wang Z, Huang Y, Pan W. Identification and characterization of novel microRNAs from Schistosoma japonicum. PLoS One. 2008; 3(12): e4034.

32. Stroehlein AJ, Young ND, Korhonen PK, Hall RS, Jex AR, Webster BL, et al. The small RNA complement of adult Schistosoma haematobium. PLoS Negl Trop Dis. 2018; 12(5): e0006535.

33. Lee Y, Han J, Yeom KH, Jin H, Kim VN. Drosha in primary microRNA processing. Cold Spring Harb Symp Quant Biol. 2006; 71: 51-7.

34. Nagy Z, Igaz P. Introduction to microRNAs: biogenesis, action, relevance of tissue microRNAs in disease pathogenesis, diagnosis and therapy-the concept of circulating microRNAs. Exp Suppl. 2015; 106: 3-30.

35. Iwasaki YW, Siomi MC, Siomi H. PIWI-interacting RNA: its biogenesis and functions. Annu Rev Biochem. 2015; 84: 405-33.

36. Mallory A, Vaucheret H. Form, function, and regulation of ARGONAUTE proteins. Plant Cell. 2010; 22(12): 3879-89.

37. Parker JS. How to slice: snapshots of Argonaute in action. Silence. 2010; 1(1): 3.

38. Zheng Y, Cai X, Bradley JE. microRNAs in parasites and parasite infection. RNA Biol. 2013; 10(3): 371-9.

39. Lee Y, Ahn C, Han J, Choi H, Kim J, Yim J, et al. The nuclear RNase III Drosha initiates microRNA processing. Nature. 2003; 425(6956): 415-9.

40. Gracheva E, Dus M, Elgin SC. Drosophila RISC component VIG and its homolog Vig2 impact heterochromatin formation. PLoS One. 2009; 4(7): e6182.

41. Huang J, Hao P, Chen H, Hu W, Yan Q, Liu F, et al. Genomewide identification of Schistosoma japonicum microRNAs using a deep-sequencing approach. PLoS One. 2009; 4(12): e8206.

42. Simões MC, Lee J, Djikeng A, Cerqueira GC, Zerlotini A, da Silva-Pereira RA, et al. Identification of Schistosoma mansoni microRNAs. BMC Genomics. 2011; 12: 47.

43. Hao L, Cai P, Jiang N, Wang H, Chen Q. Identification and characterization of microRNAs and endogenous siRNAs in Schistosoma japonicum. BMC Genomics. 2010; 11: 55.

44. Marco A, Kozomara A, Hui JH, Emery AM, Rollinson D, Griffiths-Jones S, et al. Sex-Biased expression of microRNAS in Schistosoma mansoni. PLoS One. 2013; 7(9): e2402.

45. Adema CM, Hillier LW, Jones CS, Loker ES, Knight M, Minx $\mathrm{P}$, et al. Whole genome analysis of a schistosomiasis-transmitting freshwater snail. Nat Commun. 2017; 8: 15451.

46. Zhang YQ, Chen DL, Tian HF, Zhang BH, Wen JF. Genome-wide computational identification of microRNAs and their targets in the deep-branching eukaryote Giardia lamblia. Comput Biol Chem. 2009, 33(5): 391-6.

47. Cardoso TCS, Portilho LG, de Oliveira CL, McKeown PC, Maluf WR, Gomes LA, et al. Genome-wide identification and in silico characterization of microRNAs, their targets and processing pathway genes in Phaseolus vulgaris L. Plant Biol (Stuttg). 2016; 18(2): 206-19.

48. Cardoso TCS, Alves TC, Caneschi CM, Santana DDRG, Fernandes-Brum CN, Reis GLD, et al. New insights into tomato microRNAs. Sci Rep. 2018; 8(1): 16069.

49. Lai EC, Tomancak P, Williams RW, Rubin GM. Computational identification of Drosophila microRNA genes. Genome Biol. 2003; 4(7): R42. 
50. Lagos-Quintana M, Rauhut R, Meyer J, Borkhardt A, Tuschl T. New microRNAs from mouse and human. RNA. 2003; 9(2): 175-9.

51. Cheng G, Luo R, Hu C, Cao J, Jin Y. Deep sequencing-based identification of pathogen-specific microRNAs in the plasma of rabbits infected with Schistosoma japonicum. Parasitology. 2013; 140(14): 1751-61.

52. Gomes MS, Donoghue MT, Muniyappa M, Pereira RV, Guerra-Sá $\mathrm{R}$, Spillane C. Computational identification and evolutionary relationships of the microRNA gene cluster miR-71/2 in protostomes. J Mol Evol. 2013; 76(6): 353-8.

53. Skalsky RL, Vanlandingham DL, Scholle F, Higgs S, Cullen BR. Identification of microRNAs expressed in two mosquito vectors, Aedes albopictus and Culex quinquefasciatus. BMC Genomics. 2010; 11: 119 .
54. Marco A, Hooks K, Griffiths-Jones S. Evolution and function of the extended miR-2 microRNA family. RNA Biol. 2012; 9(3): 242-8.

55. Lee RC, Ambros V. An extensive class of small RNAs in Caenorhabditis elegans. Science. 2001; 294(5543): 862-4.

56. Xu MJ, Liu Q, Nisbet AJ, Cai XQ, Yan C, Lin RQ, et al. Identification and characterization of microRNAs in Clonorchis sinensis of human health significance. BMC Genomics. 2010; 11: 521.

57. Li X, Carthew RW. A microRNA mediates EGF receptor signaling and promotes photoreceptor differentiation in the Drosophila eye. Cell. 2005; 123(7): 1267-77.

58. Bravo-Egana V, Rosero S, Molano RD, Pileggi A, Ricordi C, Domínguez-Bendala J, et al. Quantitative differential expression analysis reveals miR-7 as major islet microRNA. Biochem Biophys Res Commun. 2008; 366(4): 922-6. 